\title{
Reaction Order Parameters of Erythrocyte Sedimentation Rate, Neutrophil-to-Lymphocyte Ratio, and Platelet-to-Lymphocyte Ratio as Markers for Colorectal Carcinoma After Chemotherapy
}

\author{
Ngadikun $^{1, *}$, Untung Widodo ${ }^{2}$, Agus Barmawi ${ }^{3}$, Ahmad Hamim ${ }^{1}$, Kuncoro Asih \\ Nugroho $^{4}$, Iska Rahman Kurniawan ${ }^{5}$, Metamalik Pasala ${ }^{5}$, Izzati Amayazifun Khalifa \\ Farzana $^{6}$ \\ ${ }^{1}$ Department of Biochemistry, Faculty of Medicine Universitas Gadjah Mada Yogyakarta, Indonesia \\ ${ }^{2}$ Department of Anaesthesia and Intensive Care, Faculty of Medicine Universitas Gadjah Mada Yogyakarta, \\ Indonesia \\ ${ }^{3}$ Department of Digestive Surgery Department, Faculty of Medicine Universitas Gadjah Mada Yogyakarta, Indonesia \\ ${ }^{4}$ Department of Physics Education, Faculty of Mathematics and Natural Sciences Yogyakarta State University, \\ Indonesia \\ ${ }^{5}$ Faculty of Pharmacy Universitas Gadjah Mada, Yogyakarta, Indonesia \\ ${ }^{6}$ PKU Muhammadiyah Delanggu Hospital, Central Java, Indonesia \\ *Corresponding author. Email: ngadikun.1960@ugm.ac.id
}

\begin{abstract}
Colorectal Cancer (CRC) ranks 3rd highest cancer incidence, so it is a serious health problem in Indonesia. A new erythrocyte sedimentation rate method was developed to determine a biomarker for CRC. This study aims to investigate reaction order parameters of erythrocyte sedimentation rate (ESR), neutrophil-to-lymphocyte ratio (NLR), and platelet-to-lymphocyte ratio (PLR) as markers for colorectal carcinoma after chemotherapy. One ml blood was collected from 49 subjects by venipuncture, which then drawn for Westergren analysis. The ESR was measured every minute for 2 hours. The parameter- 1 and parameter- 2 reaction order of the two groups of research subjects were determined from ESR linear equation. The respective values of parameter-1, parameter-2, NLR, and PLR from normal group and CRC group were compared by $t$ test. The results showed that the parameters of reaction order, NLR, and PLR have good performance to be used as markers for CRC after chemotherapy.
\end{abstract}

Keywords: Colorectal cancer (CRC) Biomarker, erythrocyte sedimentation rate, neutrophil-to-lymphocyte ratio, platelet-to-lymphocyte ratio.

\section{INTRODUCTION}

Colorectal cancer (CRC) is "one of the major causes of cancer associated mortality"[1]. CRC is a multifactorial disease involving dietary, lifestyle and environment exposure as well as genetic defects [2]. Therefore, it is important to detect CRC as early as possible so treatment decisions can be made before it develops to later stage. A previous study suggested that routine screening may reduce the number of CRC patients [3], but the rates of CRC screening remain low [3] [4]. Screening methods for colorectal cancer include fecal-occult blood test and colonoscopy [5], yet the standard procedure is colonoscopy. However, colonoscopy's performance may vary depends on the operator or endoscopist. Furthermore, it requires bowel preparation [6] and sometimes sedation [7]. In addition, there is time when screening likely outweighs the benefits [8].

To overcome those problems, biomarkers are used to detect CRC. Some of the common markers in colorectal cancer are CEA, CA19-9 and CA72-4. However, these markers are not specific for cancer, as they can be found in other conditions [9]. In conclusion, the accuracy of the 
tumor markers to predict specific GI cancers in a cheap and effective way was low, so other markers are needed as alternative to overcome that problem [2].

Cancer causes chronic inflammation, which has been reported to be a crucial factor in cancer progression and cancer associated survival [10] [11]. Thus, neutrophil-tolymphocyte ratio (NLR) and platelet-to-lymphocyte ratio (PLR) as indicators of systemic inflammation are potentially used to be markers of inflammation especially in cancer [12]. Various cancer types, including gastric cancer can be predicted with an increased NLR level [13] [14]. PLR is also an inflammation indicator as Platelet releases inflammatory mediators such as cytokines and chemokines [15].

The current method cannot detect the early stages of cancer due to the low level of released proteins as biomarkers [16] [17]. Therefore, measuring the change of erythrocyte sedimentation was introduced as a new method for early detection of CRC cancer [14]. Erythrocyte sedimentation rate (ESR) is the most commonly ordered laboratory investigation as it helps clinicians in the diagnosis and follow-up of many conditions including malignancies. ESR measurement is a process when anti-coagulated blood is left stand still in room temperature so that the erythrocyte will soon aggregates to the bottom of the tube. The rate of erythrocyte sedimentation relies on may factors, with plasma protein being one of them [18]. The presence of plasma proteins increases rouleaux formation and favors to erythrocyte sedimentation [13] [19].

Cancer biomarker is commonly protein, which affects the equilibrium of erythrocyte aggregation by changing the zeta potential of erythrocyte (ZPE) in blood-EDTA [20] [21]. It will cause the decreasing of zeta potential around erythrocyte thus makes erythrocyte can easily forms rouleaux and sediment faster [22]. Fibrinogen is one of the important pro-aggregate factors in plasma. The increasing fibrinogen level will eventually decrease zeta potential further, accelerating the rate of rouleaux formation then elevating the erythrocyte sedimentation rate [18].

Protein markers are basically regular protein, which are generally amino acid residues. Each amino acid has different charges that unique to each type [23]. Negatively charged erythrocytes tend to prevent themself from colliding against each other [24]. If there is an increase in positively charged particles, the positive ions will dissipate to the surface of erythrocyte lipid bilayer membrane thus decreases repulsive force (zeta potential) of erythrocyte [25]. When the zeta potential is decreased, the repulsive force between $\mathrm{RBC}$ become weak thus will lead to aggregation process [14].

The commonly used ESR measurement has been the Westergren method, which takes the boundary between the clear (plasma regions) and cloudy (corpuscles) areas in the blood. These differential areas was resulted from the application of anticoagulants to the blood, which was kept for 1 hour on the Westergren tube. The ESR assessment is widely used due to cost-effectivity and simple use although it is relatively low in sensitivity and less specific in its activity to monitor diseases. Thus, it becomes a feasible alternative to novel measure [26]. The commonly used anti-coagulant is Ethylene Diamine Tetra Acetic Acid (EDTA), which influences the plasmarelated activity of the dissolved protein. This activity prevents the formation of erythrocytes coagulation [27]. To improve the ESR performance using the Westergren method, a method development was carried out by comparing ESR reaction order parameters of CRC group with normal group.

\section{MATERIAL AND METHODS}

\subsection{Materials}

According to the calculation, the required number of subjects is 33 for each group [28]:

$n_{1}=n_{2}=\frac{\left(z \alpha / 2+z_{\beta}\right)^{2} \times 2 \sigma^{2}}{\left(\mu_{2}-\mu_{2}\right)^{2}}$

in which $n$ denotes the number of sample in a group; $\sigma 2$ refers to the standard deviation; $\mu 1$ is the population mean time to union in intramedullary nail without reaming; $\mu 2$ is the population mean time to union intramedullary with reaming; and $\mu 1-\mu 2$ is the minimum important difference to detect in population mean time to union between group 1 and group 2. The $\alpha$ is set to 0.05 while the $\beta$ is set to 0.2 .

The subjects of this study were the CRC patients in RSUP Dr. Sardjito, both inpatients and outpatients. They were 15 to 60 year-old CRC patients who were diagnosed CRC positive from a histopathological examination. They should not have suffered from more than one types of cancer or having more than one types of cancer or any other diseases. The healthy subjects were patients with no history of cancers, no sepsis and inflammation. Their family should not have cancer history either. All participants agreed to participate in the study by signing the consent form prior to their participation. The Medical and Health Ethical Committee of the Faculty of Medicine, Gadjah Mada University (UGM) has approved the ethics (No: KE/FK/888/EC/2015).

\subsection{Methods}

This study was a cross-sectional study. It was conducted in Dr. Sardjito Hospital and Biochemistry Laboratory, Faculty of Medicine, Universitas Gadjah Mada, Yogyakarta, Indonesia in May 2015 to December 2015. The ESR method was applied to observe the cellular interaction pattern. Three $\mathrm{mL}$ Blood samples were drawn from the vein of CRC patients at the hospital 
and the healthy subjects at the laboratory. A hematology analyzer XE 2100 (Sysmex Corporation, Kobe, Japan) was used for the complete blood count (including NLR and PLR). One $\mathrm{mL}$ was placed into EDTA tubes to examine cellular interaction with ESR method while 2 $\mathrm{mL}$ was used to monitor the patient routine. The changes in the phylogistica zone boundary (bzp) were observed every 1 minute for 2 hours.

The principle of this research is to detect the "marker" of CRC patients in the form of reaction order parameters. Reaction order can be obtained by entering the numbers of all variables into the 0th to 10th reaction order equation, using the equation for each reaction order. Once entered, a linear equation for each subject in all reaction orders is obtained. From each reaction order equation, the value of parameter-1, parameter-2, and the value of the coefficient of determination ( $r$ ) of the reaction can be obtained. Based on the $r$ value, the reaction order for each subject can be determined whether it is 0 th, $1 \mathrm{st}, 2 \mathrm{nd}$, or the other. The $\mathrm{r}$ value closest to 1 or -1 is the value closest to the straight line, which means it is the closest fitting to the subject's ESR curve, so it can be used to determine the reaction order of the subject's ESR. After finding the reaction order, the value of parameter- 1 and parameter- 2 of the reaction can be determined from the values $\mathrm{B}$ and $\mathrm{C}$ of the reaction order equation.

The $\mathrm{t}$ test was carried out to see the difference between the CRC group and the normal one, by comparing the parameter values 1 and 2 of the two test groups. The t test was also carried out to compare the respective PLR and NLR values of the two groups. The significant level was taken in the $P$ value below 0.05 . The effect of cancer biomarker was evaluated using the value was determined based on the maximum sensitivity and specificity score as the optimal standard. Sensitivity and specificity value were calculated under the cut-off.

\section{RESULTS}

There were $17 \mathrm{CRC}$ patients and 32 normal subjects who became subjects of this study. The age range of the CRC group and normal group was similar. The majority of sex in both groups were women. The majority of CRC patients from this study were patients with advanced tumor stage (stage III and IV). More detailed information can be seen from Table I.

Table 1. Basic Characteristic of Study Subject

\begin{tabular}{|c|c|c|c|c|}
\hline \multirow{2}{*}{$\begin{array}{l}\text { Characteristic } \\
\text { S }\end{array}$} & \multirow{2}{*}{$\begin{array}{c}\text { CRC } \\
\text { Patient } \\
\text { S } \\
(\mathrm{N}=17)\end{array}$} & \multirow{2}{*}{$\begin{array}{c}\text { Normal } \\
\text { Subject } \\
\text { s } \\
(\mathbf{N}=32) \\
\end{array}$} & \multicolumn{2}{|c|}{ Tumor stage } \\
\hline & & & $\begin{array}{c}\text { Stag } \\
\mathrm{e}\end{array}$ & $\begin{array}{c}\text { Percentag } \\
\text { e }(\%)\end{array}$ \\
\hline Range & $\begin{array}{l}(38- \\
59) \\
\text { years }\end{array}$ & $\begin{array}{l}(19-52) \\
\text { years }\end{array}$ & I & 5.88 \\
\hline Male & $47.06 \%$ & $43.75 \%$ & II & 11.76 \\
\hline Female & $52.94 \%$ & $56.25 \%$ & III & 23.53 \\
\hline & & & IV & 23.53 \\
\hline & & & V & 35.29 \\
\hline
\end{tabular}

The mean parameters values can be seen in Table II. When compared with the normal group, it was found that all parameters are significantly different $(p<0,05$ for all parameters tested). The sensitivity and specificity values for all parameters were also shown in Table II. From the results, it can be concluded that all parameters have great performance, except specificity for PLR $(41,2 \%)$.

Table II. The Value and Performance of Reaction Order Parameters, NLR, and PLR in CRC Group Compared to Normal Group

\begin{tabular}{|c|c|c|c|c|c|c|c|c|c|c|c|}
\hline \multirow[t]{2}{*}{ Parameter } & \multicolumn{2}{|c|}{ CRC } & \multicolumn{2}{|c|}{ Normal } & \multicolumn{2}{|c|}{$\begin{array}{c}\text { Confidence Interval } \\
\text { range }\end{array}$} & \multirow{2}{*}{$\begin{array}{l}\text { Cut- } \\
\text { off } \\
\text { point }\end{array}$} & \multirow[t]{2}{*}{$\mathbf{p}$} & \multirow[t]{2}{*}{$\mathbf{A U C}$} & \multirow[t]{2}{*}{ Sensitivity } & \multirow[t]{2}{*}{$\begin{array}{l}\text { Specific } \\
\text { ity }\end{array}$} \\
\hline & average & SD & $\begin{array}{c}\text { Averag } \\
\text { e }\end{array}$ & SD & Lowest & Highest & & & & & \\
\hline Parameter- & 54.4823 & 13.2 & 41.8406 & 2.34 & 0.7366 & 0.9803 & 44.33 & 3.1 & 0.858 & 90.6 & 70.6 \\
\hline 1 & 5 & 5704 & 3 & $\begin{array}{l}903 \\
7\end{array}$ & & & 75 & $\begin{array}{l}7 \mathrm{E}- \\
06\end{array}$ & 5 & & \\
\hline Parameter- & 232.058 & 91.8 & 281.937 & 49.7 & 0.5868 & 0.8948 & 247.3 & 0.0 & 0.740 & 75 & 70.6 \\
\hline 2 & 8 & 0242 & 5 & $\begin{array}{l}295 \\
5 \\
\end{array}$ & & & & $\begin{array}{l}168 \\
85 \\
\end{array}$ & 8 & & \\
\hline NLR & $\begin{array}{l}18.1588 \\
2\end{array}$ & $\begin{array}{l}16.3 \\
6353\end{array}$ & $\begin{array}{l}3.80312 \\
5\end{array}$ & $\begin{array}{l}4.10 \\
511\end{array}$ & 0.6732 & 0.9481 & 7.25 & $\begin{array}{l}2.0 \\
8 \mathrm{E}- \\
05 \\
\end{array}$ & $\begin{array}{l}0.810 \\
7\end{array}$ & 84.4 & 76.5 \\
\hline PLR & $\begin{array}{l}4.29411 \\
8\end{array}$ & $\begin{array}{l}4.71 \\
7318\end{array}$ & 0.775 & $\begin{array}{l}0.88 \\
718 \\
5 \\
\end{array}$ & 0.6591 & 0.9401 & 3.87 & $\begin{array}{l}1.5 \\
2 \mathrm{E}- \\
04 \\
\end{array}$ & $\begin{array}{l}0.799 \\
6\end{array}$ & 100 & 41.2 \\
\hline
\end{tabular}

SD: Standard Deviation; NLR: Neutrophil-to-lymphocyte Ratio; PLR: Platelet-to-lymphocyte Ratio; AUC: Area Under Curve; NLR: Neutrophil-

receiver operating characteristic (ROC) curves. The evaluation of the biomarkers as reflected in CRC was evaluated on the area under the ROC curve (AUC) with a correspondence interval (CI) of $95 \%$. The best cut-off

\section{DISCUSSION}

Colorectal cancer (CRC) is one of gastrointestinal malignancy that contribute to $10 \%$ of all deaths caused by cancer [29]. Colorectal cancer is an adenocarcinoma 
which happens due to alteration in colonic cell's genes that causes cells to continuously proliferate and become severely dysplastic, thus lead to an invasive gastrointestinal malignancy [8]. The incidence of CRC peaks at 60 to 70 years of age [30].

Biomarkers from blood-based protein have been simpler diagnostic modalities for CRC, but their association with clinical pathological characteristics has not been evidenced. The use of blood biomarkers as a non-invasive diagnostic approach for CRC detection has been reported in a number of studies. The glycoprotein carcinoembryonic antigen (CEA) has been used widely as the blood-based CRC molecular marker for patient monitoring [31], and is firstly known in gastrointestinal carcinomas [32]. The CEA value increased with cancer progression [33]. Beside CEA, carbohydrate Antigen 199 (CA19) is also a widely used marker, but its elevation does not only happen in colorectal cancer, so it is considered to be less sensitive marker in CRC [32]. Moreover, given that the nature of $\mathrm{CRC}$ is highly heterogeneous, a single tumor marker is unlikely to be a stand-alone diagnostic test due to the insufficient sensitivity and/or specificity [31].

Carcinogenesis is a multistep process, with one of the important roles in this process is uncontrolled systemic inflammation [34]. Colorectal cancer itself is associated with the occurrence of inflammatory bowel disease. Unlike inflammatory mediators such as cytokines and chemokines, PLR and NLR are routinely examined, so they have the potential to be markers of inflammation, particularly in cancer. The inflammatory response is characterized by the increased level of neutrophils and decreased level of lymphocytes in blood. Lymphocytes have anti-inflammatory activity, and Platelets can be a non-specific marker of inflammation [12].

Neutrophil-to-lymphocyte Ratio (NLR) is the ratio of the neutrophils count divided by lymphocytes. Neutrophils and lymphocytes are indicators of systemic inflammation which are an important part of cancer development [35]. Platelet-to-lymphocyte Ratio (PLR) is the ratio of the platelet count divided by lymphocytes. The exact mechanism of platelets' effect on cancer is remain unclear, but some possible mechanisms are that the elevation of platelets can protect cancer cells from the immune system, trigger the attachment of tumor cells to the vascular endothelium, or interact with cancer cells through their ligands [34]. In addition, platelets also release pro-inflammatory mediators (such as cytokines and chemokines) [15].

NLR and PLR values are associated with tumor progression and can be used as prognostic indicators of colorectal cancers. Increased NLR and PLR values were associated with poor survival rates, possibly due to increased inflammatory activity of cancer cells [36]. The use of inflammatory indicators such as NLR and PLR has the advantage of being easier, cheaper and more convenient for patients compared to invasive methods such as biopsy because it only requires peripheral blood sampling for routine blood counts [15]. However, there are some limitations to the use of NLR and PLR, namely there are differences in threshold values from various studies, it is not specific as a marker of cancer cell malignancy [12], other inflammatory conditions can also affect the value of NLR and PLR (such as essential hypertension, acute coronary syndromes, use of drugs such as antibiotics and chemotherapy, etc) [36], and there are variations in study designs and sample sizes so that the reported results are inconsistent [37]

All reactions, both chemical reactions and physical reactions, have a reaction rate. The speed and type of reaction rate are determined by the value of the reaction order. The reaction rate curve will be different for reactions of different orders [38]. Reaction rate constant helps to determine the value of the any reaction rate [39]. The general reaction can be written as equation 2 .

$a A+b B \rightarrow d D+e E$

Base on mass reaction law, the reaction rate of $\mathrm{A}$ is explained in equation 3. Suppose $a_{0}$ is initial concentration and $\mathrm{x}$ is final concentration, so the reactant concentration change is $\left(a_{0}-x\right)$. The reaction rate of $\mathrm{A}$ is shown in equation 4 (with ' $n$ ' being the reaction order)

$v_{A}=\frac{-d[A]}{d t}\left(\frac{\mathrm{mol}}{\text { time }}\right)$

$v=\frac{-d\left(a_{0}-x\right)}{d t}=k\left(a_{0}-x\right)^{n}$

In this study, the rate of change in concentration is in the form of sedimentation length per time unit ( $\mathrm{mm} /$ minute). Thus, the value $\left(a_{0}-x\right)$ is the decrease in bzp (phlogistica zone boundary) every minute, while the value of $t$ is the time. The reduction in bzp describes the cellular interactions of red blood cells in the blood sample. The 0th order reaction can be formulated by entering the value $n=0$ into equation 4 , so it becomes equation 5 . The equation for first order reaction can also be obtained in the same way, namely by entering the value $n=1$ into equation 4 to produce equation 6 . The same process is done to get the equation for 2 nd order, 3 rd order, and so on (equation 7).

$\left(a_{0}-x\right)=-k t+C$

$\ln \left(a_{0}-x\right)=-k t+C$

$\frac{\left(a_{0}-x\right)^{1-n}}{n-1}=k t+C$

Meanwhile, the reaction order equation is a linear equation and is generally shown in equation 8 .

$Y=B x+C$

The values of $\mathrm{B}$ and $\mathrm{C}$ are the constants of linear equation, which are also called reaction order parameters. 
The value of B is the value of parameter-1, and C is the value of parameter- 2 .

The results showed that all parameters were significantly different $(p<0.05)$ with the $p$ value for Parameter-1 is 3.17E-06, Parameter-2 is 0.02 , NLR is 2.08E-05, and PLR is 1.52E-04. This shows that all parameters can differentiate between normal and CRC groups. The sensitivity and specificity values for Parameter-1 were $90.6 \%$ and $70.6 \%$, respectively; The sensitivity and specificity values for Parameter- 2 were $75 \%$ and $70.6 \%$, respectively; The sensitivity and specificity values for NLR were $84.4 \%$ and $76.5 \%$, respectively; The sensitivity and specificity values for PLR were $100 \%$ and $41.2 \%$, respectively. The sensitivity and specificity values of all these parameters are high enough that it can be concluded that all parameters have good performance as markers for CRC after chemotherapy.

Compared to the previous methods, the result of this study is more promising for three reasons. First, this study presented more comprehensive data, which included cut-off values, sensitivity and specificity, for the diagnostic research. Second, this research was one of the first studies to detect cancer without isolating biomarkers. The biomarkers in EDTA-blood affected the cellular-level interactions via ZPE changes. The Smoluchowski's theory accommodates the viscosity dispersion and dielectrics when the interaction happens[40]. As a result, a small level of biomarkers could be detected using this method. Prior to the cancer infection, the presence of one or more tumor-triggering mutations will likely result in a protein composition of the blood plasma. This process would affect ZPE. Therefore, detection of CRC biomarkers becomes more plausible with this method.

\section{CONCLUSION}

A new approach to detect biomarkers discharged by cancer cells in CRC patients has been successfully discovered, using the reaction order principle of ESR. This method is very useful because it has many advantages compared to other detection methods available, as it is cheaper, easier, faster, and only requires a small amount of blood samples, but has high sensitivity and specificity. Since it has a high sensitivity, this method can detect the presence of biomarkers in the early stages of colorectal cancer. Due to its high specificity, this method can greatly distinguish biomarkers from one another.

This method has relatively higher sensitivity and specificity than NLR and PLR. Parameter-1 has a sensitivity of $90.6 \%$, higher than NLR but still lower than PLR, and has specificity of $70.6 \%$ higher than PLR but slightly below NLR. Meanwhile, the sensitivity of Parameter-2 is $75 \%$, still lower than NLR and PLR, and specificity of $70.6 \%$, higher than PLR but slightly lower than NLR. When compared with the normal group, all parameters showed significant differences ( $\mathrm{p}<0.05)$. From these results, it can be concluded that the parameter of reaction order can be developed into a CRC diagnostic method because it has quite high sensitivity and specificity values. In addition, NLR can also be used as a parameter for the same reason, whereas PLR is less of a parameter because it has low specificity (41.2\%).

\section{ACKNOWLEDGMENTS}

The authors gratefully thank to Damas Research Grant 2015 for financial support in the study (contract number: UPPM/212/M/05/04/05.16). Special thank is also expressed for Prof. Adhi Susanto, MSc., PhD. from the Department of Electrical Engineering Faculty of Engineering, Gadjah Mada University, Yogyakarta for his support in interpreting the signal analysis of time series data. We also say thank you to RSUP dr. Sardjito for the permission given so that this research could be done, as well as to the laboratory assistant in the Biochemistry Laboratory of FKKMK UGM for their assistance during the operational of this research.

\section{REFERENCES}

[1] Krzystyniak J, Ceppi L, Dizon DS, and Birrer MJ, 2016, Epithelial ovarian cancer: the molecular genetics of epithelial ovarian cancer. Annals of Oncology, 27(1), 4-10.

[2] Song L, Jia J, Peng X, Xiao W, \& Li Y, 2017, The performance of the SEPT9 gene methylation assay and a comparison with other CRC screening tests: A meta-analysis. Scientific Reports, 7(1), 1-12.

[3] Berek JS, Crum C, and Friedlander M, 2012, Cancer of the ovary, fallopian tube, and peritoneum. Int $\mathrm{J}$ Gynecol Obstet, 119(2), 118-129.

[4] Kobayashi E, Ueda Y, Matsuzaki S, Yokoyama T, Kimura T, Yoshino K, et al, 2012, Biomarker for Screening, Diagnosis, and Monitoring of Ovarian Cancer. AACR, 12, 1902-1912.

[5] Lisi D, Hassan C, Crespi M, and AMOD Study Group, 2010, Participation in colorectal cancer screening with FOBT and colonoscopy: an Italian, multicentre, randomized population study. Digestive and liver disease, 42(5), 371-376.

[6] Romero RV and Mahadeva S, 2013, Factors influencing quality of bowel preparation for colonoscopy. World J Gastrointest Endosc, 5(2), 3946.

[7] Hüneburg R, Kukuk G, Nattermann J, Endler C, Penner A, Wolter K, et al, 2016, Colonoscopy detects significantly more flat adenomas than 3-tesla 
magnetic resonance colonography: a pilot trial. Endosc Int Open, 4, 1-6.

[8] Leggett BA and Hewett DG, 2015, Colorectal cancer screening. Intern Med J, 45, 1-10.

[9] Youssef EMI, Ewieda GH, Ali HAA and Tawfik AM, 2013, Comparison between CEA, CA 19-9 and CA 72-4 in Patients with Colon Cancer, IJTT, 2(1), 2634.

[10] Felder M, Kapur A, Gonzalez-Bosquet J, Horibata S, Heintz J, Albrecht R, et al, 2014, MUC16 (CA125): Tumor Biomarker to Cancer Therapy, a Work in Progress. Molecular Cancer, 13, 1-15.

[11] Polterauer S, Grimm C, Seebacher V, Concin N, Marth C, Tomovski C, et al, 2009, Plasma fibrinogen levels and prognosis in patients with ovarian cancer: a multicenter study. Oncologist, 14(10), 979-985.

[12] Dupré A and Malik HZ, 2018, Inflammation and cancer: What a surgical oncologist should know. European Journal of Surgical Oncology, 44(5), 566570.

[13] Ballou SP, and Kushner I, 1993, Laboratory Evaluation of Inflammation. In: Kelley WN, Harris EDJr., Ruddy S, Sledge CB, editors. Textbook of Rheumatology, 4th ed, W.B. Saunders Co: Philadelphia.

[14] Ngadikun, 1998, Pengukuran Laju Endap Darah (Erythrocyte Sedimentation Rate) dengan Metode Spektrometri, Thesis, Indonesia University: Jakarta.

[15] Kumarasamy C, Sabarimurugan S, Madurantakam RM, Lakhotiya K, Samiappan S, Baxi S, et al, 2019, Prognostic significance of blood inflammatory biomarkers NLR, PLR, and LMR in cancer - A protocol for systematic review and meta-analysis. Medicine, 98(24).

[16] Duffy MJ, Bonfrer JM, Kulpas J, Rustin GJS, Soletormos G, Torre GC, et al, 2005, CA125 in ovarian cancer: European Group on Tumor Markers guidelines for clinical use. Int J Gynecol Cancer, 15, 679-691.

[17] Fortner RT, Vitonis AF, Schock H, Hüsing A, Johnson T, Fichorova RN, et al, 2017, Correlates of circulating ovarian cancer early detection markers and their contribution to discrimination of early detection models: results from the EPIC cohort, Journal of Ovarian Research, 10, 1-14.

[18] Lichtman, M.A., Kipps, T.J., Seligsohn, U., Kaushansky, K. and Josef, T., 2011. DeVita, Hellman, and Rosenberg's Cancer Principles \& Practice 8th ed., Wolters Kluwer Health.
[19] Brahn E and Scoville CD, 1988, Biochemical Markers of Disease Activity. In: Bird H, Dixon JS, editors. Biochemical Aspects of Rheumatic Diseases. Vol. 2. Bailliere Tindall: London.

[20] Landgraf L, Christner C, Storck W, Schick I, Krumbien I, Dähring H, et al, 2015, A plasma protein corona enhances the biocompatibility of Au@Fe3O4 Janus particles, Biomaterials, 68, 77 88.

[21] Müller LK, Simon J, Schüttler S, Landfester K, Mailänder V, and Mohr K, 2016, Pre-coating with protein fractions inhibits nano-carrier aggregation in human blood plasma. RSC Adv, 6, 96495-96509.

[22] McKenzie SB, 2010, Clinical Laboratory Hematology, 2nd ed., Pearson. Cha K, Brown E, Wilmore D, 1994. A new bioelectrical impedance method for measurement of the erythrocyte sedimentation rate. Physiol Meas, 15, 499-508.

[23] Murray RK, Granner DK, and Rodwell VW, 2006, Harper's illustrated biochemistry, 27th ed, Lange Medical Books/McGraw-Hill: New York.

[24] Fernandes HP, Cesar CL and Barjas-Castro MdeL, 2011. Electrical properties of the red blood cell membrane and immunohematological investigation. Revista Brasileira de Hematologia E Hemoterapia, 33(4), 297-301.

[25] Rodak B and Fritsma G, 2012, Hematology: Clinical Principles and Application, 4th ed, Elsevier Ltd.

[26] Tas F and Erturk K, 2017, Elevated erythrocyte sedimentation rate is associated with metastatic disease and worse survival in patients with cutaneous malignant melanoma. Molecular and Clinical Oncology, 7, 1142-1146.

[27] Baskurt OK and Mesiselman HJ, 2013, Erythrocyte Aggregation: Basic Aspect and Clinical Importance. Clinical Hemorheology and Microcirculation, 53, 23-37.

[28] Farrokhyar F, Reddy D, Poolman RW and Bhandari M, 2012, why perform a priori sample size calculation? Can J surg, 56, 207-213.

[29] Jemal A, Bray F and Ferlay J, 2011, Global Cancer Statistics. CA Cancer J Clin, 49(2), 69-90.

[30] Kumar V, Abbas AK and Aster JC, 2013. Colorectal carcinoma. Robbin's Basic Pathology, 9th ed., Elsevier Ltd.

[31] Gao Y, Wang J, Zhou Y, Sheng S, Qian SY and Huo $X$, 2018. Evaluation of Serum CEA, CA19-9, CA72-4, CA125 and Ferritin as Diagnostic Markers and Factors of Clinical Parameters for Colorectal Cancer. Scientific Reports, 8(1), 1-9. 
[32] Wang J, Wang X, Yu F, Chen J, Zhao S, Zhang D, et al, 2015, Combined detection of preoperative serum CEA, CA19-9 and CA242 improve prognostic prediction of surgically treated colorectal cancer patients. IJCEP, 8(11), 14853-14863.

[33] Thomas DS, Fourkala EO, Apostolidou S, Gunu R, Ryan A, Jacobs I, et al, 2015, Evaluation of serum CEA, CYFRA21-1 and CA125 for the early detection of colorectal cancer using longitudinal preclinical samples. British Journal of Cancer, 113(2), 268-274.

[34] Li W, Tao L, Lu M and Xiu D, 2018, Prognostic role of platelet-to-lymphocyte ratio in pancreatic cancers. Medicine, 97(8), 2-8.

[35] Gürağaç A and Demirer Z, 2016, The neutrophil-tolymphocyte ratio in clinical practice. Canadian Urological Association journal, 10(3-4), 141.

[36] Yodying H, Matsuda A, Miyashita M, Matsumoto S, Sakurazawa N, Yamada M, et al, 2016, Prognostic Significance of Neutrophil-to-Lymphocyte Ratio and Platelet-to-Lymphocyte Ratio in Oncologic Outcomes of Esophageal Cancer: A Systematic Review and Meta-analysis. Annals of Surgical Oncology, 23(2), 646-654.

[37] Zheng J, Cai J, Li H, Zeng K, He L, Fu H, et al, 2017. Neutrophil-to-lymphocyte Ratio and Platelet-tolymphocyte Ratio as Prognostic Predictors for Hepatocellular Carcinoma Patients with Various Treatments: A Meta-Analysis and Systematic Review. Cell Physiol Biochem, 44(3), 967-981.

[38] Nyarugwe V, 2018, A Kinetic Study of Potassium Iodide with Potassium Persulfate using Iodine Clock Reaction. Int J Chem Sci. 16(4), 295

[39] Huang X, Zhang L, Wang X, and Wu D, 2016, Determination of the Reaction Rate Constant for the Opposing Reaction by Integral Method Determination of the reaction rate constant for the opposing reaction by integral method, Chemical Engineering Transactions, 55, 19-24.

[40] Sze A, Erickson D, Ren L, and Li D, 2003, Zetapotential measurement using the Smoluchowski equation and the slope of the current-time relationship in electroosmotic flow. J Colloid Interface Sci, 261, 402-410. 